\title{
Genotypic and Phenotypic Characteristics of Hereditary Leiomyomatosis and Renal Cell Cancer Syndrome in Korean Patients
}

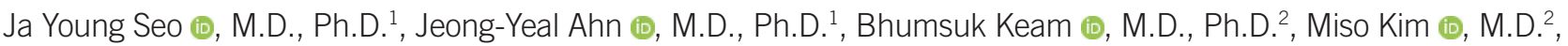

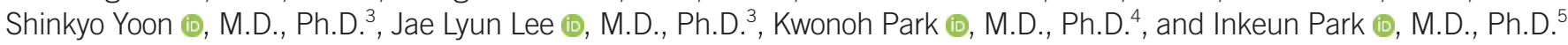

${ }^{1}$ Department of Laboratory Medicine, Gil Medical Center, Gachon University College of Medicine, Incheon, Korea; ${ }^{2}$ Division of Medical Oncology, Department of Internal Medicine, Seoul National University Hospital, Seoul, Korea; ${ }^{3}$ Department of Oncology, Asan Medical Center, University of Ulsan College of Medicine, Seoul, Korea; ${ }^{4}$ Division of Medical Oncology and Hematology, Department of Internal Medicine, Pusan National University Yangsan Hospital, Yangsan, Korea; ${ }^{5}$ Division of Medical Oncology, Department of Internal Medicine, Gil Medical Center, Gachon University College of Medicine, Incheon, Korea

Background: Hereditary leiomyomatosis and renal cell cancer (HLRCC) is an autosomal dominant cancer predisposition syndrome. HLRCC is characterized by the development of cutaneous leiomyomas, early-onset uterine leiomyomas, and HLRCC-associated renal cell cancer (RCC) and caused by germline fumarate hydratase (FH) deficiency. We investigated the genotypic and phenotypic characteristics of Korean patients with HLRCC.

Methods: We performed direct sequencing analysis of $F H$ in 13 patients with suspected HLRCC and their family members. A chromosomal microarray test was performed in female patients with negative sequencing results but highly suspected HLRCC. In addition, we analyzed the clinical characteristics and evaluated the genotype-phenotype correlations in Korean patients with HLRCC.

Results: We identified six different pathogenic or likely pathogenic $F H$ variants in six of the 13 patients (46.2\%). The variants included two nonsense variants, two splicing variants, one frameshift variant, and one missense variant. Of the six variants, two (33.3\%) were novel (c.132+1G>C, and c.243dup). RCC and early-onset uterine leiomyoma were frequently observed in families with HLRCC, while cutaneous leiomyoma was less common. No significant genotype-phenotype correlation was observed.

Conclusions: We describe the genotypic and phenotypic spectrum in a small series of Korean patients with HLRCC. Our data reveal the unique characteristics of Korean patients with HLRCC and suggest a need for establishing an optimal diagnostic approach for them.

Key Words: FH, Genotype, Hereditary leiomyomatosis and renal cell cancer, Korean, Novel variant, Phenotype
Received: February 19, 2020

Revision received: March 30, 2020

Accepted: September 19, 2020

\section{Corresponding author: \\ Ja Young Seo, M.D., Ph.D. \\ Department of Laboratory Medicine, Gil \\ Medical Center, Gachon University College \\ of Medicine, 21 Namdong-daero 774 \\ Beon-gil, Namdong-gu, Incheon 21565, \\ Korea \\ Tel: +82-32-460-3832 \\ Fax: +82-32-460-3415 \\ E-mail: jyseojy@gilhospital.com}

\section{Co-corresponding author: \\ Inkeun Park, M.D., Ph.D. \\ Division of Medical Oncology, Department of Internal Medicine, Gachon University Gil Medical Center, 21 Namdong-daero 774 \\ Beon-gil, Namdong-gu, Incheon 21565, \\ Korea \\ Tel: +82-32-460-3229 \\ Fax: +82-32-460-2391 \\ E-mail: ingni79@gilhospital.com}

\section{(i) (2)}

(c) Korean Society for Laboratory Medicine This is an Open Access article distributed under the terms of the Creative Commons Attribution Non-Commercial License (https://creativecommons.org/licenses/by-nc/4.0) which permits unrestricted non-commercial use, distribution, and reproduction in any medium, provided the original work is properly cited.

\section{INTRODUCTION}

Hereditary leiomyomatosis and renal cell cancer (HLRCC; MIM 150800 ) is a rare autosomal dominant cancer predisposition syndrome characterized by the variable development of cutaneous leiomyomas, early-onset uterine leiomyomas (fibroids), and type 2 papillary renal cell cancer (RCC), which is now classified separately as HLRCC-associated RCC [1, 2]. HLRCC is caused 
by germline loss of function variants in the $\mathrm{FH}$ gene, which encodes the Krebs cycle protein fumarate hydratase (FH) [3]; $F H$ pathogenic variants have been identified in approximately 70$90 \%$ of individuals with suspected HLRCC [4-6]. Over 200 families with HLRCC have been reported worldwide [4-11]; however, the prevalence of HLRCC is unknown, and it may be underdiagnosed because of insufficient awareness of the condition and incomplete penetrance of HLRCC-associated tumors, especially RCC [6]. HLRCC-associated RCCs are found in only $20 \%-34 \%$ of affected individuals, while cutaneous and uterine leiomyomas occur in approximately $70-100 \%$ of patients with HLRCC by 30 years of age [12]. To date, no genotype-phenotype correlation has been identified for HLRCC [4, 6, 13].

In Korea, HLRCC and HLRCC-associated RCC have been only recently recognized, and data regarding the treatment outcome of HLRCC-associated RCC in a small series of genetically confirmed Korean HLRCC patients were reported in 2019 [10, 11]. We thus investigated the genetic spectrum of FH pathogenic variants in Korean patients with HLRCC, their phenotypic characterization, and the genotype-phenotype correlation. To the best of our knowledge, this is the first study to explore the clinical and genetic characteristics of Korean patients with HLRCC.

\section{MATERIALS AND METHODS}

\section{Study patients}

This study included Korean patients with suspected HLRCC at four academic hospitals in Korea (Gil Medical Center, Asan Medical Center, Seoul National University Hospital, and Pusan National University Yangsan Hospital) between December 2017 and August 2019. The selection criteria for the germline $F H$ tests were as follows: histologically confirmed skin leiomyomatosis, suggestion of HLRCC-associated RCC based on clinicopathologic characteristics (young onset, non-clear cell RCC, including type 2 papillary RCC, a family history of RCC, very rapid progress, and unresponsiveness to standard treatments), early-onset symptomatic uterine leiomyomas in female patients, and a family history of HLRCC-associated symptoms. Five patients were recruited from a previous study by Choi, et al. [10] (Table 1). Demographic and clinical characteristics including age, sex, HLRCC-related phenotypes, and family history of participants were prospectively collected. Family members of genetically confirmed HLRCC patients were tested for germline variants of the FH gene regardless of symptoms.

This study was approved by the Institutional Review Board of Gachon University Gil Medical Center, Incheon, Korea (GAIRB 2017-365). Written informed consent for the study was obtained from all participants.

Table 1. Clinical characteristics and $F H$ variants of the 13 probands

\begin{tabular}{|c|c|c|c|c|c|c|}
\hline \multirow{2}{*}{ Case $\mathrm{N}$} & \multirow{2}{*}{ Sex } & \multirow{2}{*}{$\begin{array}{c}\text { RCC } \\
\text { (age, yr) }\end{array}$} & \multirow{2}{*}{$\begin{array}{l}\text { Hysterectomy or } \\
\text { myomectomy (age, yr) }\end{array}$} & \multirow{2}{*}{ Family history } & \multicolumn{2}{|c|}{ FHvariant } \\
\hline & & & & & Identified variant & ACMG classification \\
\hline $1^{*}$ & M & Yes (46) & NA & Uterine leiomyoma, cutaneous lesion & c. $1108+1 G>T$ & PV \\
\hline 2 & $\mathrm{~F}$ & Yes (40) & Yes (40) & Uterine leiomyoma & c. $634 C>T\left(p . G \ln 212^{\star}\right)$ & PV \\
\hline $3^{*}$ & M & Yes (42) & NA & No & $c .132+1 G>C$ & PV \\
\hline $4^{*}$ & M & Yes (29) & NA & RCC, uterine leiomyoma, cutaneous lesion & c.688A > G (p.Lys230Glu) & LPV \\
\hline 5 & M & Yes (60) & NA & No & ND & \\
\hline $6^{*}$ & M & Yes (42) & NA & RCC, uterine leiomyoma & c.1027C > T (p. $\left(\operatorname{Arg} 343^{\star}\right)$ & PV \\
\hline 7 & M & Yes (34) & NA & № & ND & \\
\hline 8 & $\mathrm{~F}$ & Yes (64) & Yes (44) & Uterine leiomyoma & ND & \\
\hline 9 & $\mathrm{~F}$ & Yes (24) & No & № & ND & \\
\hline $10^{*}$ & M & Yes (49) & NA & № & c.842C > T (p.Thr281Ile) & VUS \\
\hline 11 & $\mathrm{~F}$ & Yes (30) & Yes (30) & Uterine leiomyoma & ND & \\
\hline 12 & $\mathrm{~F}$ & No & Yes (27) & RCC & c243dup (p.Gly82Trpfs*13) & PV \\
\hline 13 & $\mathrm{~F}$ & Yes (25) & Yes (25) & RCC, uterine leiomyoma & ND & \\
\hline
\end{tabular}

*Patients included in the study by Choi, et al. [10].

Novel variants are shown in italics.

Abbreviations: ACMG, American College of Medical Genetics and Genomics; M, male; F, female; FH, fumarate hydratase; PV, pathogenic variant; LPV, likely pathogenic variant; NA, not applicable; ND, not detected; RCC, renal cell cancer; VUS, variant of uncertain clinical significance. 


\section{Molecular genetic testing}

Fresh peripheral blood samples were obtained and transported, under refrigerated conditions if necessary, to the molecular diagnostic laboratory in Gil Medical Center within 24 hours. We performed direct sequencing of the $\mathrm{FH}$ gene, including all coding exons and flanking intronic regions, using genomic DNA extracted from peripheral blood leukocytes (QIAamp DSP DNA Mini Kit; Qiagen, Valencia, CA, USA). Polymerase chain reaction (PCR) was performed using in-house designed primers (Supplemental Data Table S1). Direct sequencing was performed on an Applied Biosystems 3500xL Genetic Analyzer (Applied Biosystems, Foster City, CA, USA) using a BigDye Terminator v3.1 Cycle Sequencing Kit (Applied Biosystems). Sequence variants were identified based on GenBank reference sequences NM_ 000143.3 and NP_000134.2 using Sequencher 4.9 (Gene Codes Corporation, Ann Arbor, MI, USA). The identified sequence variants were interpreted and classified according to the 2015 American College of Medical Genetics and Genomics standards and guidelines [14]. Chromosomal microarray (CMA) was performed for female patients with negative sequencing analysis results who had both type 2 papillary RCC and uterine leiomyoma with a probable family history, using the CytoScan Dx Assay test with a resolution of $400 \mathrm{~kb}$ (Affymetrix, Santa Clara, CA, USA).

\section{RESULTS}

\section{Clinical characteristics of the patients}

The study included 13 probands (seven males and six females) and 19 family members. The demographic and clinical characteristics of the probands are presented in Table 1. Except one, they (12/13, 92.3\%) were diagnosed as having RCC (nine papillary type, one mixed papillary and clear cell type, one clear cell type, and one unclassified). One female proband (P12) underwent a hysterectomy at 27 years of age, and a family history of type 2 papillary RCC was documented for her (in her father). Eight of the $13(61.5 \%)$ probands had a family history of HL-
RCC-associated symptoms. Four of the six female probands had both RCC and uterine leiomyomas, and three of these underwent a hysterectomy before the age of 40 years. In addition, three of the seven (42.9\%) male probands had female family members with a history of early-onset uterine leiomyomas. None of the patients in our case series had all three representative HLRCC tumors.

\section{FH genetic testing}

We found six different pathogenic or likely pathogenic $F H$ variants in six of the 13 probands (46.2\%) and one variant of uncertain clinical significance (VUS) in one proband (7.7\%). The pathogenic or likely pathogenic variants included two nonsense variants, two splicing variants, one frameshift variant, and one missense variant; two (33.3\%) of these variants were novel (Table 1). We found a novel missense variant in P10 [c.842C > T (p.T281I)], which was previously reported by Choi, et al. [10]. However, we classified the variant as VUS because it was also detected in three asymptomatic family members. All of these variants were located in the lyase domain (Fig. 1). Five genetically confirmed HLRCC probands (all except P3) had a family history of HLRCC-associated tumors. No significant variant was

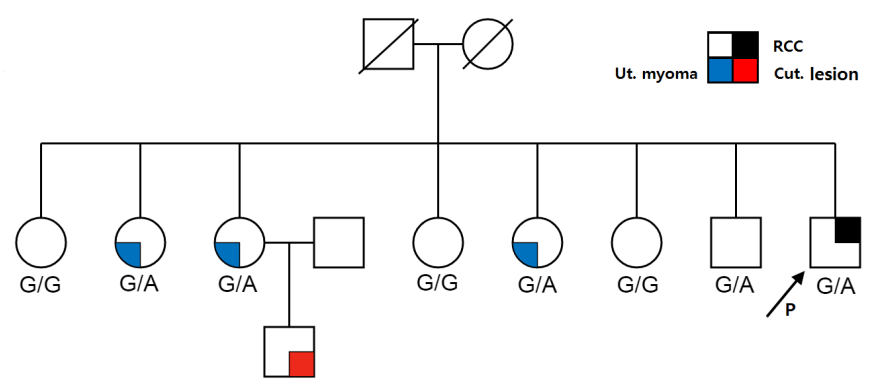

Fig. 2. Pedigree of $P 1$ harboring the c.1108+1G $>A$ variant (indicated by arrow). All three sisters of $P 1$ who are carriers of the $c .1108+1 G>A$ variant underwent a hysterectomy before the age of 40 years. $F H$ genetic testing was not performed for the son of the third sister. P1's older brother was asymptomatic at the time of genetic testing. Abbreviations: Ut, uterine; Cut, cutaneous.

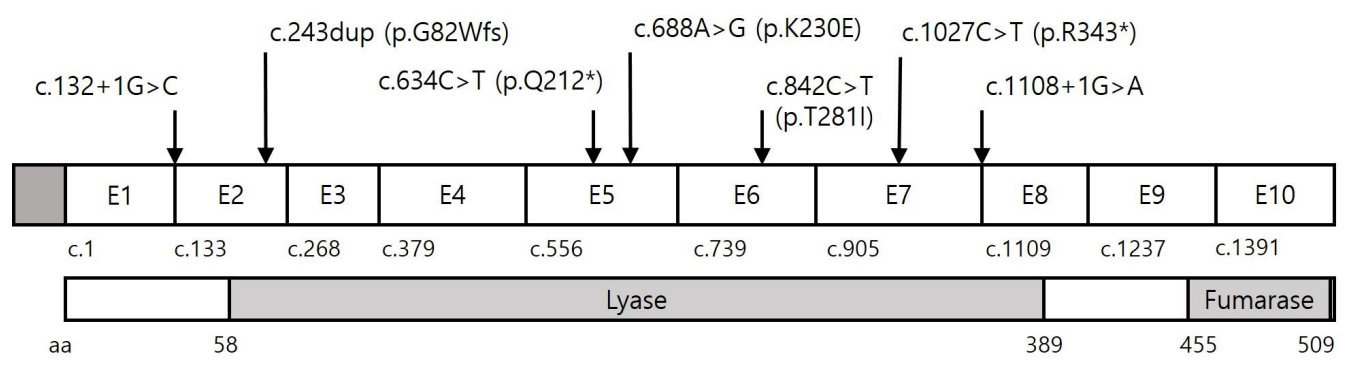

Fig. 1. Distribution of $F H$ variants identified in this study. 
identified by direct sequencing analysis in six probands. Of these, three female probands (P8, P11, and P13) had both type 2 papillary RCC and uterine leiomyomas with a probable family history; however, no significant copy number variation (CNV) was detected by CMA testing. The other three probands had isolated type 2 papillary RCC without a definite family history.

We performed a targeted variant testing in 18 family members of the three probands with pathogenic $F H$ variants (seven for $\mathrm{P} 1$, four for $\mathrm{P} 3$, and one for P12) and the proband with the VUS (six for P10) and found pathogenic variants in five of them. Of the seven family members of $P 1$, three female carriers had undergone a hysterectomy before the age of 40 years (Fig. 2). The remaining two family members with a pathogenic $F H$ variant (49-year-old brother from P1 and 24-year-old sister from P13) were asymptomatic at the time of genetic testing. Three of the six family members of P10 (with the VUS) had the same variant without any clinical symptoms. The detailed clinical and genetic characteristics of the family members who underwent targeted variant testing are described in Supplemental Data Table S2.

\section{Phenotypic characterization and genotype-phenotype correlation}

All pathogenic and likely pathogenic variants were associated with RCC. Early-onset uterine leiomyomas were documented in all families with pathogenic or likely pathogenic $F H$ variants, except in one proband (P3) whose mother and four sisters had neither HLRCC-associated symptoms nor a pathogenic $F H$ variant. Cutaneous leiomyoma-like lesions were noted in the family members of P1 (c.1108+1G >A) and P4 [c.688A > G (p.K230E)], although a pathological diagnosis was not performed [11]. No significant genotype-phenotype correlation was observed.

\section{DISCUSSION}

We identified six families with HLRCC and six pathogenic $F H$ variants, two of which were novel variants. Type 2 papillary RCC and early-onset uterine leiomyomas were frequently observed in the carriers of $\mathrm{FH}$ pathogenic variants, whereas cutaneous leiomyomas were relatively uncommon in our case series. In addition, we did not identify a definitive genotype-phenotype correlation.

In 2011, Smit, et al. [8] proposed criteria for the clinical diagnosis of HLRCC; according to these, diagnosis is likely when a proband has histopathologically confirmed multiple cutaneous piloleiomyomas (major criterion) or meets at least two of the following minor criteria: (1) surgical treatment for symptomatic uterine leiomyomas before the age of 40 years, (2) development of type 2 papillary RCC before the age of 40 years, and (3) has a first-degree family member who meets one of these criteria. Smit, et al. [8] observed 100\% penetrance of cutaneous lesions in their study group aged $>40$ years; however, none of the probands in our case series met the major criterion. All female carriers in our study underwent a hysterectomy before the age of 40 years, except one 24-year-old asymptomatic carrier, while only two probands were diagnosed as having type 2 papillary RCC before the age of 40 years (median 42 years; range, 29-46 years). Choi, et al. [10] reported that the median age of RCC diagnosis in 10 Korean HLRCC patients was 41 years (range, 2752 years). Although Caucasian data have shown a high penetrance of cutaneous leiomyomas in HLRCC, such penetrance was less prevalent in Korean patients [5, 6, 8, 13]. Similarly, cutaneous manifestations were absent in Japanese and Chinese HLRCC patients [15-17]. It is likely that the phenotypic spectrum of HLRCC differs between Asian and Caucasian populations. There is a possibility of under-ascertainment of cutaneous leiomyomas as the participants of the current study mainly included patients with RCC who were recruited from the oncology department. It is also possible that our probands did not include patients with isolated uterine or cutaneous leiomyomas (formerly known as multiple cutaneous and uterine leiomyomatosis or Reed's syndrome).

In the female carriers of $\mathrm{FH}$ pathogenic or likely pathogenic variants, uterine leiomyomas developed before the age of 40 years, with the earliest onset at 27 years of age. Early-onset uterine leiomyomas were the only manifestation noted in four of the seven female carriers at the time of the genetic testing. Uterine leiomyomas are the most common benign neoplasm in the female reproductive system; therefore, the condition itself is not likely to be taken into account when diagnosing HLRCC. To identify women with uterine leiomyomas who are at increased risk of HLRCC, pathology-based screening of uterine leiomyomas has been suggested, including $\mathrm{FH}$-deficiency (FH-d) morphology (smooth muscle tumor cells with macronucleoli surrounded by a halo and cytoplasmic eosinophilic globules) and FH immunohistochemical staining [18-20]. Rabban, et al. [20] reported that $60 \%$ of women with an FH-d morphology had germline $\mathrm{FH}$ variants, and $0.24 \%$ of all women with any type of uterine leiomyoma were diagnosed as having HLRCC. In Korea, HLRCC has only recently been recognized by medical oncologists, while gynecologists and pathologists are unfamiliar with HLRCC-associated uterine leiomyomas. Therefore, our data demonstrate the necessity of pathology-based identification of candidates for HLRCC genetic testing among Korean women with early-onset uter- 
ine leiomyomas, which would enable clinical and radiological surveillance of HLRCC-associated RCC.

HLRCC-associated RCC has distinctive papillary architecture characteristics; however, none of the patients in our study were initially diagnosed as having HLRCC-associated RCC by a pathologist, as it is unknown to pathologists in Korea. In addition to a papillary pattern, a broad spectrum of architectural patterns has been reported for HLRCC-associated RCC, exclusive of clear cell type [7, 21, 22]. Therefore, ancillary tests, such as FH and 2-succinocystein immunohistochemical staining or a somatic/ germline variant test, will be helpful [7]. In a study of germline testing in patients with advanced RCC unselected for inherited syndrome risk factors, $\mathrm{FH}$ variants were the most common RCCassociated variants (seven of 41) [23].

Currently, approximately 100 pathogenic or likely pathogenic FH variants are listed in the ClinVar database (accessed December 2019). The variants include 78 null variants and 21 missense variants. Unlike the Caucasian data, which indicated that more than a half of the variants were missense variants, five of the six (83.3\%) pathogenic/likely pathogenic variants in our study were null variants $[4,7,13]$. Large deletions, including that of the entire $F H$ gene, have been identified in previous studies [3, 13, 24, 25]; however, we did not find any significant CNV in the three female patients who were highly suspected as having HLRCC based on the CMA test. Nevertheless, the possible presence of small exonal deletions cannot be ruled out as the detection limit of the CMA test used in this study was $400 \mathrm{~kb}$. Additional highresolution molecular tests, such as real-time PCR, multiplex ligation-dependent probe amplification, and high-definition arraycomparative genomic hybridization, are needed to detect small CNVs. It is possible that the $F H$ sequencing-negative patients have other inherited cancer syndromes predisposing them to RCC, such as von Hippel-Lindau syndrome, hereditary papillary RCC, Birt-Hogg-Dubé syndrome, and tuberous sclerosis, although RCCs associated with these syndromes display distinctive clinicopathological characteristics [26, 27]. RCC predisposition gene panel tests may help identify patients with germline variants of cancer-susceptibility genes for whom tailored therapies and cancer screenings are indicated.

Our detection rate of $\mathrm{FH}$ pathogenic/likely pathogenic variants was relatively lower than those observed in previous studies (46.2\%; 6/13; Table 2). The selection criteria we used for the germline FH tests were less strict than those used by Smit, et al. [8], indicating that patients less likely to have HLRCC were enrolled. Contrarily, our data showed that the HLRCC diagnosis criteria proposed by Smit, et al. [8] are less relevant to Korean patients,
Table 2. Frequency of $F H$ PV/LPVs in various populations

\begin{tabular}{lcc}
\hline Reference & $\begin{array}{c}\text { Geographic distribution/ } \\
\text { Ethnic background }\end{array}$ & $\begin{array}{c}\text { Frequency of FH PV/LPV, } \\
\%(\mathrm{~N})\end{array}$ \\
\hline Toro, et al. (2006) [5] & North America & 93 (52/56 families) \\
Gardie, et al. (2011) [6] & France & 55.7 (44/79 families)* \\
Alam, et al. (2003) [7] & United Kingdom & $76.1(35 / 46)$ \\
Smit, et al. (2011) [8] & The Netherlands & $42.4(14 / 33$ families) \\
Bhola, et al. (2018) [9] & Canada & $69.5(48 / 69)$ \\
Present study & Korea & $46.2(6 / 13)$
\end{tabular}

*The frequency of FH PV/LPV was 71.4\% (40/56) in suspected HLRCC families and $17.4 \%(4 / 23)$ in isolated type 2 papillary RCC families.

Abbreviations: $F H$, fumarate hydratase; LPV, likely pathogenic variant; PV, pathogenic variant; HLRCC, hereditary leiomyomatosis and renal cell cancer; RCC, renal cell cancer.

as none of the patients in our study met the major criterion, histopathologically confirmed multiple cutaneous piloleiomyomas. Thus, we suggest that $F H$ genetic testing should be considered in Korean patients (1) who have non-clear cell RCC (especially with a type 2 papillary pattern) with a family history of RCC or early-onset uterine leiomyomas and (2) female patients with earlyonset ( $<40$ years old) uterine leiomyomas involving multiple family members, regardless of the presence of RCC and/or cutaneous leiomyomas.

This study has potential limitations; it included a small number of patients from only four academic hospitals. Further, no central pathology review was conducted. Finally, sequencingnegative cases were not scrutinized using alternative molecular methodologies.

In summary, we describe the genotypic and phenotypic spectrum in a small series of Korean patients with HLRCC. Our data reveal the unique characteristics of Korean HLRCC patients, and the very low cutaneous leiomyoma penetrance. In addition, our data highlight the need for a more comprehensive approach for diagnosing patients with RCC and uterine/cutaneous leiomyomas who may benefit from tailored treatment and appropriate surveillance for cancer. Further studies on other Korean patients with HLRCC are required to delineate the clinical characteristics and establish a diagnostic strategy.

\section{ACKNOWLEDGEMENTS}

None.

\section{AUTHOR CONTRIBUTIONS}

IP, JLL, BK, and JYS conceived, initiated, and designed the 
study. IP and JYS performed data analysis and wrote the manuscript. BK, MK, SY, JLL, KP, and IP were responsible for interview, informed consent, and data collection from the study participants. JYS and JYA performed genetic analyses. MK, SY, and $\mathrm{KP}$ supervised the study design, and reviewed and commented on the manuscript. All authors read and approved the final manuscript.

\section{CONFLICTS OF INTEREST}

No potential conflicts of interest relevant to this paper were reported.

\section{RESEARCH FUNDING}

None declared.

\section{ORCID}

Ja Young Seo
Jeong-Yeal Ahn
Bhumsuk Keam
Miso Kim
Shinkyo Yoon
Jae-Lyun Lee
Kwonoh Park
Inkeun Park

https://orcid.org/0000-0002-1894-1365
https://orcid.org/0000-0001-9842-0748
https://orcid.org/0000-0001-8196-4247
https://orcid.org/0000-0002-4064-4199
https://orcid.org/0000-0001-7544-0404
https://orcid.org/0000-0002-9420-7162
https://orcid.org/0000-0002-7404-1257
https://orcid.org/0000-0003-3064-7895

\section{REFERENCES}

1. Launonen V, Vierimaa O, Kiuru M, Isola J, Roth S, Pukkala E, et al. Inherited susceptibility to uterine leiomyomas and renal cell cancer. Proc Natl Acad Sci U S A 2001;98:3387-92.

2. Moch H, Cubilla AL, Humphrey PA, Reuter VE, Ulbright TM. The 2016 WHO classification of tumours of the urinary system and male genital organs-part A: renal, penile, and testicular tumours. Eur Urol 2016;70: 93-105.

3. Tomlinson IP, Alam NA, Rowan AJ, Barclay E, Jaeger EE, Kelsell D, et al. Germline mutations in $\mathrm{FH}$ predispose to dominantly inherited uterine fibroids, skin leiomyomata and papillary renal cell cancer. Nat Genet 2002;30:406-10.

4. Toro JR, Nickerson ML, Wei MH, Warren MB, Glenn GM, Turner ML, et al. Mutations in the fumarate hydratase gene cause hereditary leiomyomatosis and renal cell cancer in families in North America. Am J Hum Genet 2003;73:95-106.

5. Wei MH, Toure O, Glenn GM, Pithukpakorn M, Neckers L, Stolle C, et al. Novel mutations in $\mathrm{FH}$ and expansion of the spectrum of phenotypes expressed in families with hereditary leiomyomatosis and renal cell cancer. J Med Genet 2006;43:18-27.

6. Gardie B, Remenieras A, Kattygnarath D, Bombled J, Lefevre S, PerrierTrudova $\mathrm{V}$, et al. Novel $\mathrm{FH}$ mutations in families with hereditary leiomyo- matosis and renal cell cancer (HLRCC) and patients with isolated type 2 papillary renal cell carcinoma. J Med Genet 2011;48:226-34.

7. Alam NA, Rowan AJ, Wortham NC, Pollard PJ, Mitchell M, Tyrer JP, et al. Genetic and functional analyses of $F H$ mutations in multiple cutaneous and uterine leiomyomatosis, hereditary leiomyomatosis and renal cancer, and fumarate hydratase deficiency. Hum Mol Genet 2003;12: 1241-52.

8. Smit DL, Mensenkamp AR, Badeloe S, Breuning MH, Simon ME, van Spaendonck KY, et al. Hereditary leiomyomatosis and renal cell cancer in families referred for fumarate hydratase germline mutation analysis. Clin Genet 2011;79:49-59.

9. Bhola PT, Gilpin C, Smith A, Graham GE. A retrospective review of 48 individuals, including 12 families, molecularly diagnosed with hereditary leiomyomatosis and renal cell cancer (HLRCC). Fam Cancer 2018;17: 615-20.

10. Choi Y, Keam B, Kim M, Yoon S, Kim D, Choi JG, et al. Bevacizumab plus erlotinib combination therapy for advanced hereditary leiomyomatosis and renal cell carcinoma-associated renal cell carcinoma: a multicenter retrospective analysis in Korean patients. Cancer Res Treat 2019; 51:1549-56.

11. Park I, Shim YS, Go H, Hong BS, Lee JL. Long-term response of metastatic hereditary leiomyomatosis and renal cell carcinoma syndrome associated renal cell carcinoma to bevacizumab plus erlotinib after temsirolimus and axitinib treatment failures. BMC Urol 2019;19:51.

12. Patel VM, Handler MZ, Schwartz RA, Lambert WC. Hereditary leiomyomatosis and renal cell cancer syndrome: an update and review. J Am Acad Dermatol 2017;77:149-58.

13. Muller M, Ferlicot S, Guillaud-Bataille M, Le Teuff G, Genestie C, Deveaux $S$, et al. Reassessing the clinical spectrum associated with hereditary leiomyomatosis and renal cell carcinoma syndrome in French $\mathrm{FH}$ mutation carriers. Clin Genet 2017;92:606-15.

14. Richards S, Aziz N, Bale S, Bick D, Das S, Gastier-Foster J, et al. Standards and guidelines for the interpretation of sequence variants: a joint consensus recommendation of the American College of Medical Genetics and Genomics and the Association for Molecular Pathology. Genet Med 2015;17:405-24.

15. Kuwada M, Chihara Y, Lou Y, Torimoto K, Kagebayashi Y, Tamura K, et al. Novel missense mutation in the $\mathrm{FH}$ gene in familial renal cell cancer patients lacking cutaneous leiomyomas. BMC Res Notes 2014;7:203.

16. Noguchi G, Furuya M, Okubo Y, Nagashima Y, Kato I, Matsumoto K, et al. Hereditary leiomyomatosis and renal cell cancer without cutaneous manifestations in two Japanese siblings. Int J Urol 2018;25:832-5.

17. Zhao Z, Wang W, You Y, Zhu L, Feng F. Novel FH mutation associated with multiple uterine leiomyomas in Chinese siblings. Mol Genet Genomic Med 2019:e1068.

18. Joseph NM, Solomon DA, Frizzell N, Rabban JT, Zaloudek C, Garg K. Morphology and immunohistochemistry for 2SC and $\mathrm{FH}$ aid in detection of fumarate hydratase gene aberrations in uterine leiomyomas from young patients. Am J Surg Pathol 2015;39:1529-39.

19. Siegler L, Erber R, Burghaus S, Brodkorb T, Wachter D, Wilkinson N, et al. Fumarate hydratase (FH) deficiency in uterine leiomyomas: recognition by histological features versus blind immunoscreening. Virchows Arch 2018;472:789-96.

20. Rabban JT, Chan E, Mak J, Zaloudek C, Garg K. Prospective detection of germline mutation of fumarate hydratase in women with uterine smooth muscle tumors using pathology-based screening to trigger genetic counseling for hereditary leiomyomatosis renal cell carcinoma syndrome: a 5-year single institutional experience. Am J Surg Pathol 2019;43:63955.

21. Merino MJ, Torres-Cabala C, Pinto P, Linehan WM. The morphologic 
spectrum of kidney tumors in hereditary leiomyomatosis and renal cell carcinoma (HLRCC) syndrome. Am J Surg Pathol 2007;31:1578-85.

22. Chen YB, Brannon AR, Toubaji A, Dudas ME, Won HH, Al-Ahmadie $\mathrm{HA}$, et al. Hereditary leiomyomatosis and renal cell carcinoma syndromeassociated renal cancer: recognition of the syndrome by pathologic features and the utility of detecting aberrant succination by immunohistochemistry. Am J Surg Pathol 2014;38:627-37.

23. Carlo MI, Mukherjee S, Mandelker D, Vijai J, Kemel Y, Zhang L, et al. Prevalence of germline mutations in cancer susceptibility genes in patients with advanced renal cell carcinoma. JAMA Oncol 2018;4:122835.

24. Koski TA, Lehtonen HJ, Jee KJ, Ninomiya S, Joosse SA, Vahteristo P, et al. Array comparative genomic hybridization identifies a distinct DNA copy number profile in renal cell cancer associated with hereditary leiomyomatosis and renal cell cancer. Genes Chromosomes Cancer 2009; 48:544-51.

25. Vocke CD, Ricketts CJ, Merino MJ, Srinivasan R, Metwalli AR, Middelton LA, et al. Comprehensive genomic and phenotypic characterization of germline $\mathrm{FH}$ deletion in hereditary leiomyomatosis and renal cell carcinoma. Genes Chromosomes Cancer 2017;56:484-92.

26. Linehan WM, Lerman MI, Zbar B. Identification of the von Hippel-Lindau (VHL) gene. Its role in renal cancer. JAMA 1995;273:564-70.

27. Zbar B, Glenn G, Merino M, Middelton L, Peterson J, Toro J, et al. Familial renal carcinoma: clinical evaluation, clinical subtypes and risk of renal carcinoma development. J Urol 2007;177:461-5; discussion 465. 
Supplemental Data Table S1. Primer sequences used for FH genetic testing

\begin{tabular}{ll}
\hline Primer name & \multicolumn{1}{c}{ Sequence } \\
\hline FH_E1F & TGTGAGGCTGTGATTGGAT \\
FH_E1R & GGAGGGCTGAAGGTCACTG \\
FH_E2F & AAGATGCGATTACTTTGATCC \\
FH_E2R & TGAATACAGCCTACTTATCCAA \\
FH_E3F & CTGCCAAAATAATAAACTCCATGC \\
FH_E3R & GCCAGAGCATATCGTCATCC \\
FH_E4F & TGTGGGTCAACTGTATCAAAC \\
FH_E4R & CAATCTCAGGTATGCTTTCAA \\
FH_E5F & GCTGGGTTTGAGTAGTTAGTTG \\
FH_E5R & GGCCATTGTACCAAGCTCT \\
FH_E6F & CCCTCATCCTTCCCTATACTTG \\
FH_E6R & CACAAGAATTCAAGACAGGAACA \\
FH_E7F & TGGAACTTCTGTTCACTTC \\
FH_E7R & CATGGTCCATAGCTAAGAATGC \\
FH_E8F & ATGGTGGGCCTGCTTTAT \\
FH_E8R & CCAAGATAATAAGCCTTGGTCA \\
FH_E9F & CATGTGGCCTAGTAATGTCTCTCTC \\
FH_E9R & TGCTGTTCTCAAACACTGATCC \\
FH_E10F & CAATTATGTCACCTTTGCTTAGG \\
FH_E10R & GCAGTTCCTTCAAACTTATCC \\
\hline &
\end{tabular}


Seo JY, et al.

HLRCC in Korean patients

Supplemental Data Table S2. Clinical and genetic characteristics of the patients' family members

\begin{tabular}{|c|c|c|c|c|c|c|}
\hline Case N & Sex & $\begin{array}{l}\text { Age at } F H \text { gene test } \\
(y r)\end{array}$ & Relationship & $\begin{array}{c}\text { Hysterectomy or myomectomy } \\
\text { (age, yr) }\end{array}$ & $F H$ variant & Comments \\
\hline $1-1$ & $\mathrm{~F}$ & 64 & 1st sister & No & No & \\
\hline $1-2$ & $\mathrm{~F}$ & 61 & 2nd sister & 30 & $P V(c .1108+1 G>A)$ & \\
\hline $1-3$ & $\mathrm{~F}$ & 58 & 3rd sister & 39 & $\mathrm{PV}(\mathrm{c} .1108+1 \mathrm{G}>\mathrm{A})$ & Skin lesion in sor \\
\hline $1-4$ & $\mathrm{~F}$ & 55 & 4th sister & No & ND & \\
\hline $1-5$ & $\mathrm{~F}$ & 54 & 5th sister & 31 & $P V(c .1108+1 G>A)$ & \\
\hline $1-6$ & $\mathrm{~F}$ & 52 & 6th sister & No & ND & \\
\hline $1-7$ & M & 49 & Brother & NA & $P V(c .1108+1 G>A)$ & \\
\hline $3-1$ & $\mathrm{~F}$ & 63 & Mother & No & ND & \\
\hline $3-2$ & $\mathrm{~F}$ & 42 & 1st sister & No & ND & \\
\hline $3-3$ & $\mathrm{~F}$ & 40 & 2nd sister & No & ND & \\
\hline $3-4$ & $\mathrm{~F}$ & 38 & 3rd sister & No & ND & \\
\hline $3-5$ & $\mathrm{~F}$ & 35 & 4th sister & No & ND & \\
\hline $10-1$ & M & Unknown & Father & NA & ND & \\
\hline $10-2$ & $\mathrm{~F}$ & Unknown & Mother & No & $\operatorname{VUS}(c .842 \mathrm{C}>\mathrm{T})$ & \\
\hline $10-3$ & M & Unknown & 1st brother & NA & VUS (c.842C > T) & \\
\hline $10-4$ & M & Unknown & 3rd brother & NA & ND & \\
\hline $10-5$ & $\mathrm{~F}$ & Unknown & Niece & No & $\operatorname{VUS}(c .842 \mathrm{C}>\mathrm{T})$ & \\
\hline $10-6$ & M & Unknown & Nephew & NA & ND & \\
\hline $13-1$ & $\mathrm{~F}$ & 24 & Sister & No & PV (c.243dup) & \\
\hline
\end{tabular}

Abbreviations: M, male; F, female; FH, fumarate hydratase; NA, not applicable; ND, not detected; PV, pathogenic variant; RCC, renal cell cancer; VUS, variant of uncertain clinical significance. 